\title{
Delay measurement of attosecond emission in solids
}

\author{
Dong Hyuk Ko ${ }^{1} \odot$, Graham G Brown ${ }^{1}$, Chunmei Zhang ${ }^{1}$ \\ and $\mathbf{P}$ B Corkum ${ }^{1,2,3}$ \\ 1 Joint Attosecond Science Laboratory, Department of Physics, University of Ottawa, Ottawa, ON K1N \\ 6N5, Canada \\ 2 Joint Attosecond Science Laboratory, National Research Council of Canada, Ottawa, ON K1A 0R6, \\ Canada \\ E-mail:dko@uottawa.ca,gbrown093@uottawa.ca,Chunmei.Zhang@uottawa.ca and \\ pcorkum@uottawa.ca
}

Received 21 January 2020, revised 27 February 2020

Accepted for publication 20 March 2020

Published 27 May 2020

\begin{abstract}
We characterize an attosecond pulse without using a secondary laser pulse. Instead, we use the carrier-envelope-phase dependence of the extreme ultraviolet (XUV) emission to measure the dipole current from a solid material. To confirm, we apply our approach to data derived by solving the time-dependent Schrödinger equation with a periodic potential. Finally, using $\mathrm{MgO}$, we measure the delay shift at the strong XUV absorption feature and explain it as the quantum interference between the continuum and bound states that participate in the generation process.
\end{abstract}

Keywords: strong-field physics, attosecond pulse measurement, delay in recollision process

(Some figures may appear in colour only in the online journal)

Attosecond science has shown that extreme ultraviolet (XUV) radiation created by the recollision of highly energetic electrons accelerated by a strong laser field permits the generation of very short pulses with superb coherence [1]. Enhancing the brightness of the XUV light, tuning its frequency, and controlling the XUV spatial profiles have been an aim of attosecond science since its inception [2-4]. Broadening the bandwidth of the generated XUV and synchronizing the spectral components are important to improve the temporal resolution of attosecond pulses [5,6]. Recently, it has become possible to control the polarization of the XUV light and shape the spatial mode with higher orbital angular momentum [7, 8]. In parallel with all of the above objects, we also need to develop measurement methods available in XUV region.

Temporal characterization of attosecond pulses is essential for understanding ultrafast processes between electrons or electrons and holes [9]. Previous methods for the measurement of attosecond pulses rely on the cross-correlation of the XUV radiation and an additional laser field to achieve a two-photon transition or to perturb ionized electrons while generating high

\footnotetext{
${ }^{3}$ Author to whom any correspondence should be addressed.
}

harmonics [10-12]. In this paper, we show that an attosecond pulse can be measured without using a secondary laser field. Instead, we use the carrier-envelope-phase (CEP) dependence of the generation of XUV radiation when the driving laser pulse is very short. This is later confirmed through theoretical derivation and numerical simulations.

We will show that the narrow time window of a few-cycle pulse, essential for generating isolated attosecond pulses, can itself be used as a gating function for attosecond pulse characterization. Since the XUV emission driven by strong laser fields repeats for every half optical cycle of the driving laser pulse, a series of attosecond pulses is generated, i.e. an attosecond pulse train. Reducing the number of XUV pulses in a pulse train has been a prominent issue for the generation of isolated attosecond pulses. Temporal gating, by utilizing time-varying polarization, was proposed in 1994 and demonstrated in 2006 as one method to generate isolated attosecond pulses [13-15]. Another method involves compressing the driving laser pulse as short as possible to limit the generation of high-frequency photons to the peak of the driving laser pulse occurring in a single half cycle [16], but this requirement of a short driving pulse can be somewhat relaxed by mixing a polarization gated pulse 
with its second harmonic [17]. All of these methods have the same purpose and can be qualitatively understood as grating the XUV emission in time to a single attosecond burst.

We propose to characterize the temporal structure of an attosecond pulse by taking the narrow envelope of the fewcycle driving laser pulse itself as a gating function. One can decompose the strong driving laser pulse into two parts-a high frequency carrier and its envelope. The carrier is a sinusoidal function responsible for the creation of XUV emission in every half optical cycle. The envelope limits the number of XUV emissions in time by limiting the number of cycles in the pulse.

Before beginning, it is useful to consider a grating analogy. Each attosecond pulse within a train of pulses is similar to the diffracted light from the grooves of a grating. The gating function corresponds to the width of the grating, defining the number of diffracting grooves. The CEP determines the relative position of the attosecond pulse train with respect to the gating function, equivalent to the determining the relative position of the groove lines on the grating. When there are many lines on the grating, the diffracted spectrum is insensitive to the relative position of the groove. However, it becomes extremely sensitive when the grating has only a few illuminated lines. We will use this analogy as we apply the formalism of grating physics to the attosecond pulse measurement. We will show that the attosecond pulse and the envelope of the driving laser pulse can be measured from the CEP-dependence of the dipole spectrum.

Dipole radiation created by light-matter interaction with a strong laser field is well understood by the following equation from literature when considering the driving laser field as a continuous wave $[18,19]$ :

$$
X(t)=\int_{0}^{\infty} d \tau\left(\frac{\pi}{\xi+i \tau / 2}\right)^{1 / 2} R(t) S(t, \tau) T(t-\tau) .
$$

$T(t-\tau)$ is the ionization rate for the electron from the ground state to continuum by tunneling at $t-\tau$ (ionization time). $S(t, \tau)$ is the time evolution function of the ionized electron in the continuum from $t-\tau$ (ionization time) to $t$ (recombination or emission time) while it is accelerated by the laser field during $\tau$ (excursion time). $R(t)$ is the dipole recombination rate from the continuum to the ground state at $t$. The first factor in the $\tau$-integration originates from the 1D momentum integration with a regularized Gaussian function around the saddle point by taking into account the small value of $\xi$. We define the Fourier conjugate function for $R(t) T(t-\tau)$ as in the reference [15]:

$$
b(v, \tau)=\int_{-\infty}^{\infty} R(t) T(t-\tau) \mathrm{e}^{i v t} \mathrm{~d} t
$$

Then the spectrum of the dipole radiation is obtained by performing a Fourier transform according to the convolution theorem:

$$
X(\omega)=\int_{-\infty}^{\infty} \mathrm{d} v \int_{0}^{\infty} d \tau\left(\frac{\pi}{\xi+i \tau / 2}\right)^{1 / 2} S(\omega-v, \tau) b(v, \tau) .
$$

Now, we consider a non-uniform envelope $G\left(t-\tau-\phi / \omega_{0}\right)$ of the driving laser pulse, which limits the number of attosecond emissions in time. Here $\phi$ is the CEP and $\omega_{0}$ is the angular frequency of the driving laser field. The CEP-dependent dipole spectrum is written as the following by replacing $T(t-\tau)$ with $T(t-\tau) G\left(t-\tau-\phi / \omega_{0}\right)$

$$
\begin{aligned}
H(\omega, \phi)= & \int_{-\infty}^{\infty} \mathrm{d} t \mathrm{e}^{i \omega t} \int_{0}^{\infty} d \tau\left(\frac{\pi}{\xi+i \tau / 2}\right)^{1 / 2} R(t) \\
& \times S(t, \tau) T(t-\tau) G\left(t-\tau-\phi / \omega_{0}\right) \\
= & \int_{-\infty}^{\infty} d \Omega \mathrm{e}^{i \Omega\left(\phi / \omega_{0}\right)} G(\Omega) \int_{0}^{\infty} d \tau \mathrm{e}^{i \Omega \tau}\left(\frac{\pi}{\xi+i \tau / 2}\right)^{1 / 2} \\
& \times \int_{-\infty}^{\infty} \mathrm{d} v S(\omega-\Omega-v, \tau) b(v, \tau)
\end{aligned}
$$

Here $\Omega$ is the energy shift by the gating function $G\left(t-\tau-\phi / \omega_{0}\right)$, which is the Fourier conjugate variable for $t-\tau-\phi / \omega_{0}$. Since the factor for the wave function diffusion comes from the momentum integration of the regularized Gaussian wave packet, we can combine the first two terms in the $\tau$-integration. Note that the electron kinetic energy $p^{2} / 2$ is very large compared to $\Omega$. It allows us to change the variable of integration $p$ to $p^{\prime}$ :

$$
\begin{aligned}
\mathrm{e}^{i \Omega \tau}\left(\frac{\pi}{\xi+i \tau / 2}\right)^{1 / 2} & =\mathrm{e}^{i \Omega \tau} \int_{-\infty}^{\infty} d p \mathrm{e}^{-i \frac{p^{2}}{2} \tau} \mathrm{e}^{-\xi p^{2}} \\
& \approx \mathrm{e}^{-2 \Omega \xi} \int_{-\infty}^{\infty} d p^{\prime} \mathrm{e}^{-i \frac{p^{\prime 2}}{2} \tau-\xi p^{\prime 2}} \\
& \approx\left(\frac{\pi}{\xi+i \tau / 2}\right)^{1 / 2} \quad(\text { due to } \xi \approx 0)
\end{aligned}
$$

which follows from $p^{2}-2 \Omega \approx p^{2}$.

Consequently, the CEP-dependent dipole spectrum can be described as a cross-correlation of the dipole radiation and the gating function in frequency, which is equivalent to the principle equation used for the pulse characterization [20-23]:

$$
\begin{aligned}
H(\omega, \phi)= & \int_{-\infty}^{\infty} d \Omega \mathrm{e}^{i \Omega\left(\phi / \omega_{0}\right)} G(\Omega) \int_{0}^{\infty} d \tau\left(\frac{\pi}{\xi+i \tau / 2}\right)^{1 / 2} \\
& \times \int_{-\infty}^{\infty} \mathrm{d} v S(\omega-\Omega-v, \tau) b(v, \tau) \\
= & \int_{-\infty}^{\infty} d \Omega \mathrm{e}^{i \Omega\left(\phi / \omega_{0}\right)} G(\Omega) X(\omega-\Omega) \\
\text { where } X(\omega)= & \int_{0}^{\infty} d \tau\left(\frac{\pi}{\xi+i \tau / 2}\right)^{1 / 2} \int_{-\infty}^{\infty} \mathrm{d} v \\
& \times S(\omega-v, \tau) b(v, \tau) .
\end{aligned}
$$

To test our approach, we artificially construct $H(\omega, \phi)$ by assuming $G(\Omega)$ and $X(\omega)$, representing a gating function with a narrow window of 1.2 fs repeating by a $\pi$-CEP period and a positively chirped attosecond pulse for a bandwidth of 20 
(a)

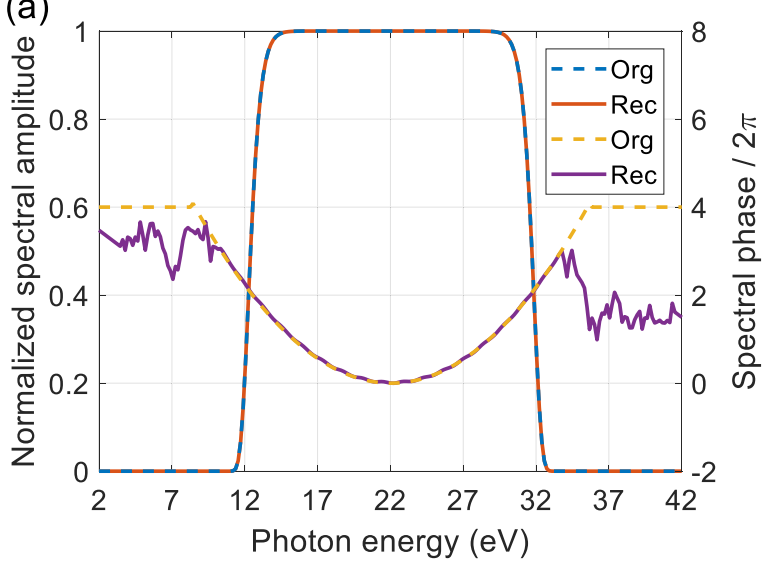

(c)

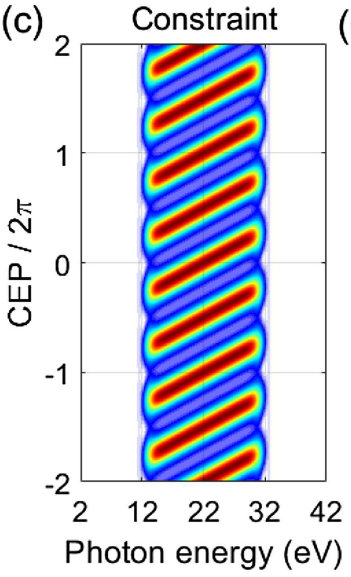

(d)

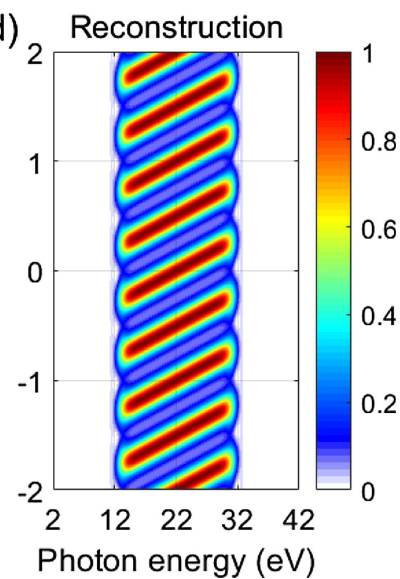

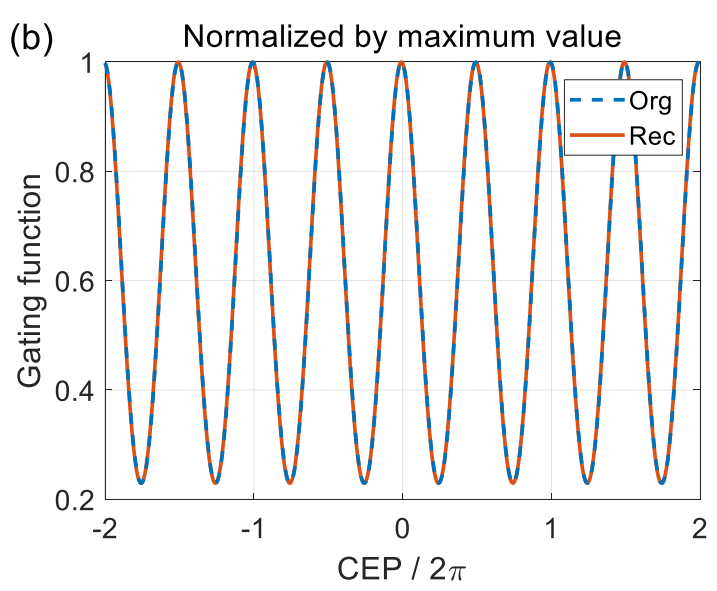

(e)

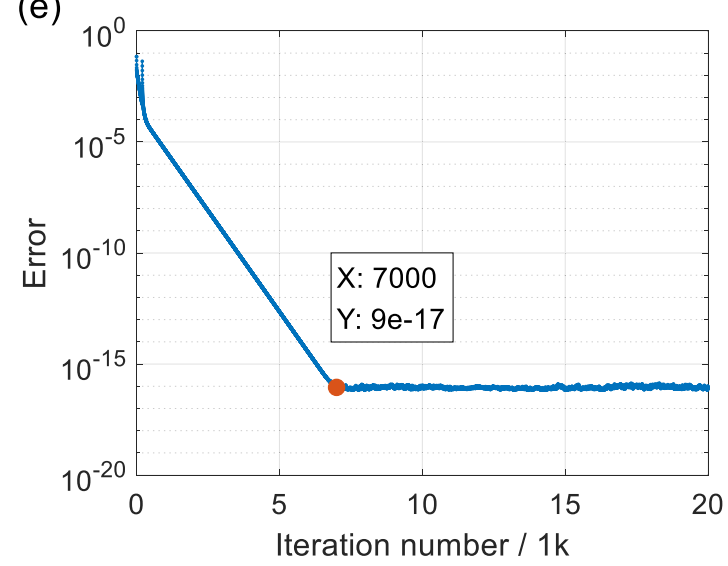

Figure 1. (a) Comparison of $X(\omega)$ for the original and retrieved spectra and phases. (b) Comparison of $G(\phi)$ for the original and retrieved functions. (c) Assigned XUV spectra as a function of CEP for the reconstruction. (d) Reconstructed CEP-dependent XUV spectra by the phase retrieval method. (e) Error progression of the phase retrieval method for the CEP-dependent XUV spectra.

$\mathrm{eV}$ around a photon energy of $22 \mathrm{eV}$ with a dispersion of $0.06 \mathrm{fs}^{2}$ [24]. Figure 1(a) shows the spectral amplitude and phase (depicted by dashed lines) of the attosecond pulse that we have constructed for the numerical simulation. $G(\phi)$, a CEP-dependent gating function, is presented in figure 1(b) by taking the Fourier transform of $G(\Omega)$. Finally, we obtain the CEP-dependent dipole spectra as shown in figure 1(c), which is used as an intensity constraint in the phase retrieval method.

We then verify that an attosecond pulse and a gating function can be measured by applying a phase retrieval algorithm to the CEP-dependent dipole spectra [25]. This is a reverse process to determine two functions, $G(\Omega)$ and $X(\omega)$, from a given two-dimensional function $H(\omega, \phi)$. Figure $1(\mathrm{~d})$ is the reconstructed $H(\omega, \phi)$ by the phase retrieval method after running 20000 iterations. The error in figure 1(e) reaches $9 \times 10^{-17}$ after 7000 iterations, monotonically decreasing exponentially. The reconstructed $X(\omega)$ and $G(\phi)$ in the figures 1(a) and (b) are nearly identical to the functions that we assumed in the beginning of the simulation.

To demonstrate our method, we now apply the method to experiment. We use the CEP-dependent dipole spectra obtained from $\mathrm{MgO}$. For the experiment, we generate a CEPstabilized two-cycle laser pulse by delivering a $5 \mathrm{~mJ} 25$ fs $800 \mathrm{~nm}$ Ti:sappire laser pulse to an optical parametric amplifier (OPA). The $1.8 \mu \mathrm{m}$ idler is passively CEP stabilized with a standard deviation of $260 \mathrm{mrad}$. This pulse is compressed in an Ar-filled hollow-core fiber where its spectrum is stretched and chirped to cover a wavelength bandwidth from 1.4-2.2 $\mu \mathrm{m}$. The pulse is then compressed by a $2 \mathrm{~mm}$-thick piece of fused silica to produce a two-cycle pulse [26]. Finally, this two-cycle laser pulse, with an energy of $100 \mu \mathrm{J}$, is focused into a $200 \mu \mathrm{m}$-thick MgO crystal, where it reflectively generates XUV radiation from the crystal surface [27].

The XUV spectrum (figure 2(a)) is measured as a function of the CEP by using an X-ray imaging spectrometer, consisting of two toroidal mirrors and an XUV grating. Due to the very short duration of the driving laser pulse, in the near cutoff region of the dipole radiation we observe CEP-modulated XUV spectra around $22 \mathrm{eV}$ with a bandwidth of $3 \mathrm{eV}$. The modulation is similar to that in the figure 1(c).

We apply our method to the measured XUV spectra of the figure 2(a) for the reconstruction of the attosecond pulse generated from the $\mathrm{MgO}$. The CEP-dependent XUV spectra (figure 2(b)) are recovered after more than 40000 iterations of the phase retrieval algorithm. The figure shows a $\pi$-CEP modulation with a slope similar to that in the figure 2(a). The reconstructed spectral amplitude and group delay of the XUV pulse generated from the $\mathrm{MgO}$ crystal are presented in figure 2(c) 

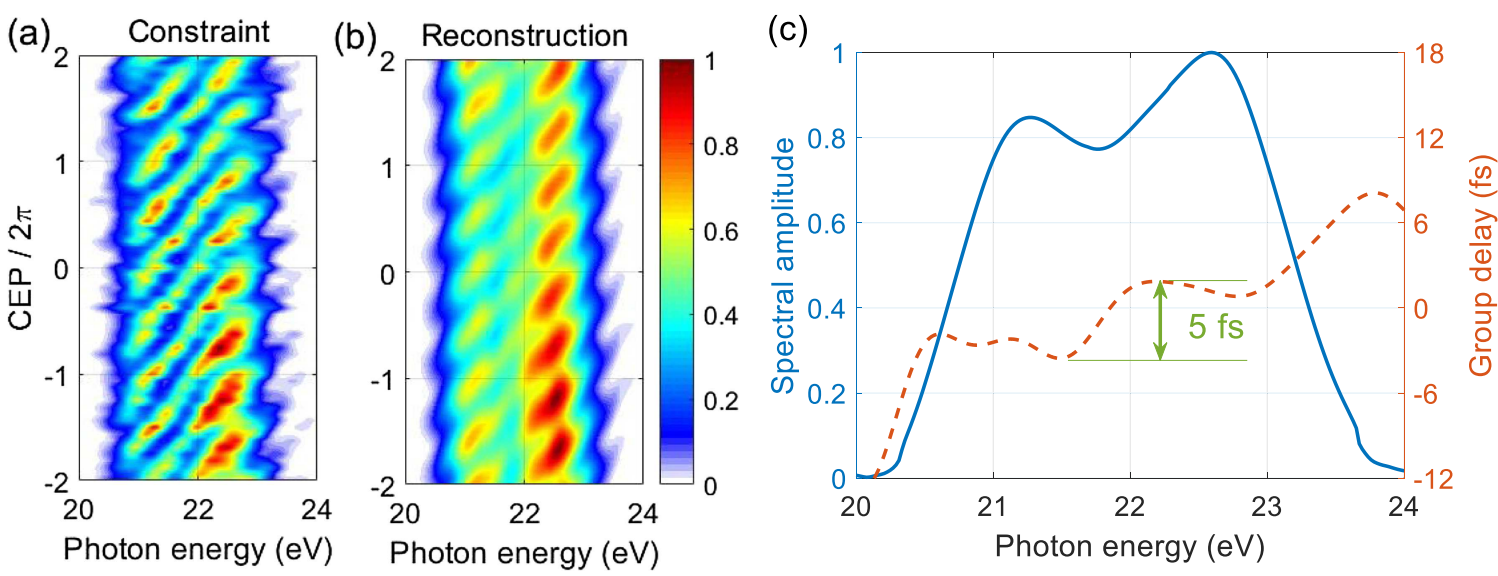

Figure 2. (a) CEP-dependent XUV spectra meausred in experiment and (b) the reconstructed result by the phase retrieval method. (c) XUV spectrum and group delay of the attosecond pulse generated from the $\mathrm{MgO}$ crystal achieved by the reconstruction.

by solid and dashed lines, respectively. The XUV spectrum has a two-peak structure due to a peak in the crystal absorption at $22 \mathrm{eV}$ and this results in a positive delay of $5 \mathrm{fs}$.

Now, to conclude the paper we turn to simulation once again. We perform a one dimensional simulation of XUV generation from a $\mathrm{MgO}$ crystal by solving the time-dependent Schrödinger equation with a periodic potential [28-30]. We consider a single active electron moving along the laser field polarization for simplicity. The periodic structure of the crystal is treated by superposing regularly positioned 64 softcore potentials with a lattice distance of $8 \mathrm{a}_{0}$ (Bohr radius). The spatial grid is composed of 2048 points with an extent of $\pm 256 \mathrm{a}_{0}$, corresponding to a momentum space extent up to $\pm 4 \pi \hbar / \mathrm{a}_{0}$ (atomic units of momentum) with a resolution of $2 \pi / 512 \hbar / a_{0}$. First we calculate the field-free eigenstates and their corresponding eigenenergies.

Figure 3(a) shows the energy levels of our crystal model in momentum space for the time-independent Hamiltonian. Energy level splitting is clearly seen in the figure 3(a). We choose the lattice distance and soft-core parameter to match the band gap energies of $\mathrm{MgO}$ between the valence band and the $1 \mathrm{st}$ and $3 \mathrm{rd}$ conduction bands, which are $7.8 \mathrm{eV}$ and $22 \mathrm{eV}$, respectively. The band gap energy between the 2 nd and $3 \mathrm{rd}$ conduction bands is $0.18 \mathrm{eV}$.

We then determine the time dependent evolution of the ground state electron wave function created by the laser field. For this, we apply a monocycle laser pulse with a wavelength of $1.8 \mu \mathrm{m}$ and peak intensity of $10^{13} \mathrm{~W} \mathrm{~cm}^{-2}$ (both similar to our experimental conditions). The time-dependent dipole current is calculated by taking the expectation value of the momentum operator. Since we already have a complete set of electron wave functions from the ground state analysis, we project the time-dependent electron wave function to each band and decompose the dipole current into inter band contributions between the 3rd conduction band and valence band.

Figure 3(b) shows the spectrum and group delay of the calculated dipole current around the XUV photon energy of $22 \mathrm{eV}$, plotted by a blue solid line and red dashed line respectively. We find a strong absorption peak near $22 \mathrm{eV}$ with 5.2 fs positive delay at the absorption energy that agrees with the corresponding absorption and delay observed experimentally, as depicted in the figure 2(c). The difference of the spectral amplitude and group delay between the experiment and calculation can be attributed to the reduced diffusion of the ionized electron wave packet for the long trajectory radiations due to the use of a single spatial dimension in the simulation. Further, instrumental broadening makes the spectrum widen and the absorption dip at the center be reduced. Due to the set up of our XUV spectrometer, the resolving power is 32 , determined by our $500 \mu \mathrm{m}$-wide slit and the corresponding energy resolution is $0.69 \mathrm{eV}$ at the photon energy of $22 \mathrm{eV}$. Therefore, we roughly estimate the linewidth of the absorption to be $0.2 \mathrm{eV}$, close to the band gap of $0.18 \mathrm{eV}$, by considering the measured bandwidth of $0.88 \mathrm{eV}$ from the figure 2(c). The results show the attosecond pulse exhibits small group delay dispersion (i.e. the slope of the group delay curve is small).

The absorption and the delay shift of $\mathrm{MgO}$ are a quantum resonance phenomena. They arise from the existence of two pathways that lead to the creation of XUV photons (figure 3(c)). An electron initially in the ground state can be ionized by the laser field, indicated by the labelled black arrow. The laser field drives the electron in the conduction band where it approaches the next band. The electron then recombines, radiating an XUV photon with the energy of $22 \mathrm{eV}$, marked in magenta. This is the regular high harmonic generation process in atoms [1]. This direct pathway for the XUV photon generation is described in the equation (7) by a delta-function in the $\tau$-integration:

$$
\begin{aligned}
X_{\text {bandgap }}(t)= & \langle g|p| k-A(t)\rangle \int_{0}^{t} d \tau[i \delta(\tau) \\
& +\langle k-A(t)|\Gamma| k-A(t-\tau)\rangle] \\
& \times \int_{0}^{t-\tau} d \tau^{\prime}\left\langle k-A(t-\tau)|S| k-A\left(t-\tau-\tau^{\prime}\right)\right\rangle \\
& \times\left\langle k-A\left(t-\tau-\tau^{\prime}\right)\left|T\left(t-\tau-\tau^{\prime}\right)\right| g\right\rangle
\end{aligned}
$$


(a)

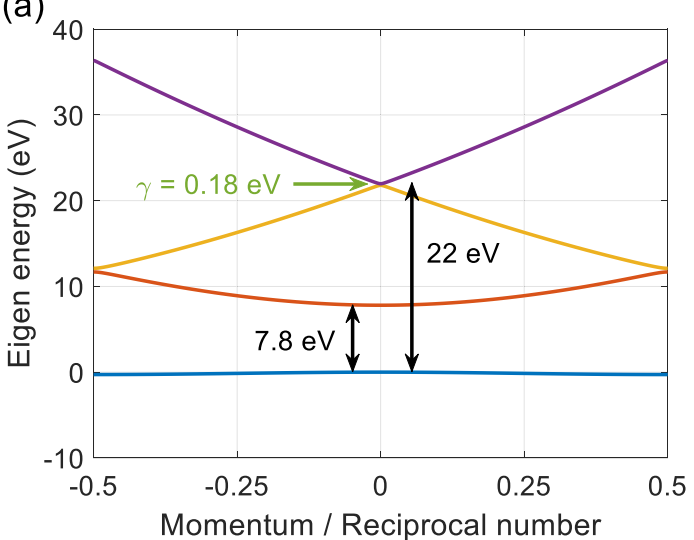

(c)

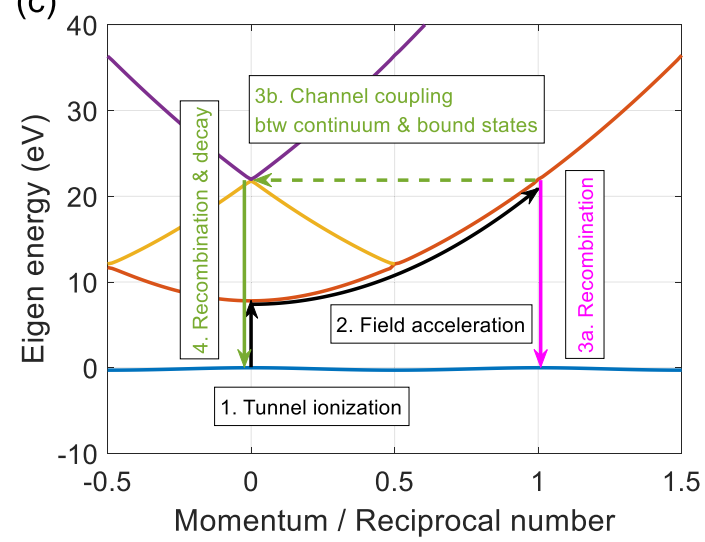

(b)

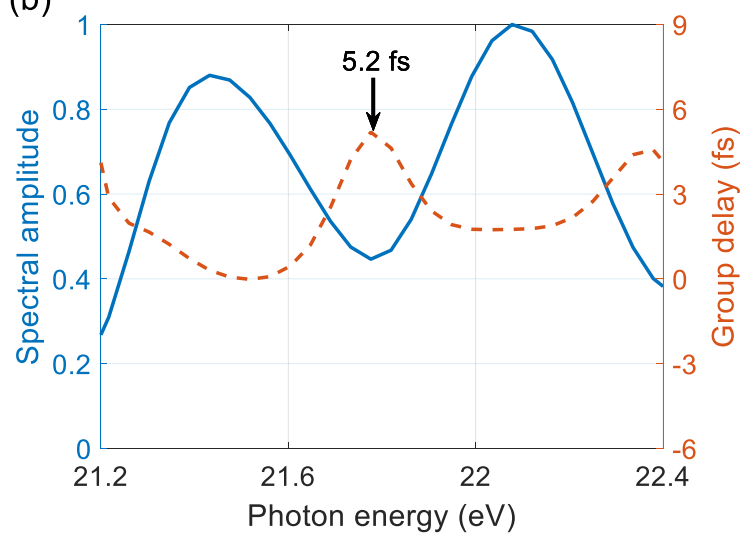

(d)

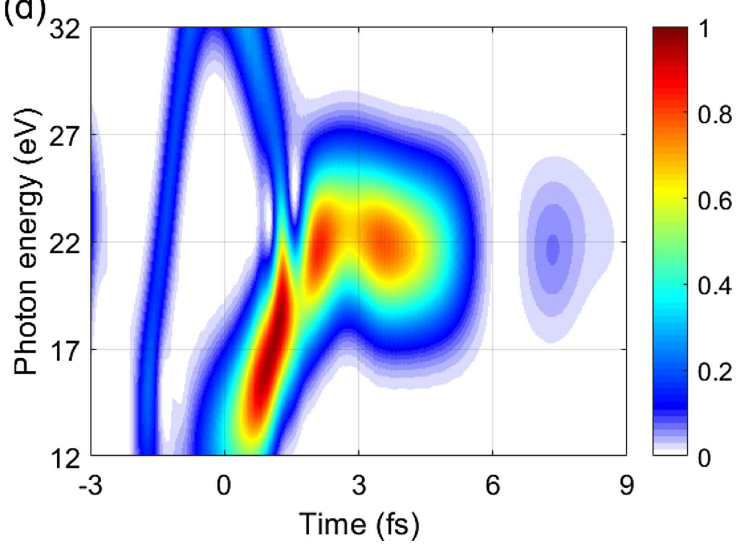

Figure 3. (a) Energy diagram of the 1D periodic potential model for simulating the MgO crystal. (b) XUV spectrum and group delay of the attosecond pulse calculated by the 1D TDSE periodic potential model. (c) Step-wise explanation for the resonance process in the MgO crystal at $22 \mathrm{eV}$. (d) Time-frequency distribution of the calculated attosecond pulse showing time-delayed tail at $22 \mathrm{eV}$ after $2 \mathrm{fs}$.

Here $|g\rangle$ and $|k\rangle$ are the eigen functions in momentum space for the hole and electron, respectively. $T$ is the time-dependent operator representing tunnel ionization and $S$ is the timeevolution operator from $t-\tau-\tau^{\prime}$ to $t-\tau$ with the vector potential for field acceleration. The last term in the time integration represents the recombination of the electron in the conduction band to the ground state creating the timedependent dipole current. Alternatively, the free electron in the continuum state can make a transition to the next highest band by tunneling through the band gap for the energy of $0.18 \mathrm{eV}$ and then return to the ground state. This is expressed in the equation (7) as the second term of the bracket in the $\tau$ integration, usually defined as an exponential decay with the decay time of $1 / \gamma$ (here the band gap $\gamma$ is $0.18 \mathrm{eV}$ ). As a result, the time-relaxed tail of the dipole current is clearly seen in figure 3(d) with its time-frequency distribution at $22 \mathrm{eV}$ after 2 fs by applying short-time Fourier transform. Due to the small contribution of long trajectory electron wave packets, negatively chirped XUV radiation above $22 \mathrm{eV}$ is weak and the radiation due to short-trajectory is dominant under the decay at $22 \mathrm{eV}$.

In conclusion, we demonstrate that attosecond pulse measurement is possible using the CEP-dependence of XUV emission when using a few cycle driver. We have derived the principle equations for our method using the strong field approximation. Then, we measure the XUV emission from
$\mathrm{MgO}$ around its band gap of $22 \mathrm{eV}$ and observe a group delay shift in a region of strong absorption and retrieve the phase of the attosecond pulse using this method.

It is well known that the shaping and engineering of attosecond pulses is possible using material dispersion in solid crystals. Compression of a positively chirped attosecond pulse necessitates the pulse travel through a medium with negative dispersion around the spectral region of interest [31]. Similarly, solid materials with periodic structure also have negative dispersion around the band gap energy due to the strong absorption by the forbidden zone as shown in the figures 2(c) and 3(b). By choosing or designing a solid state medium with tailored absorption properties, this negative dispersion can be used to compensate the intrinsic positive chirp of attosecond pulses in a given spectral range. Thus, attosecond pulses can be optimized while they are being generated from solids by combining material science and semiconductor technology and tailoring solid media for attosecond pulse generation $[32,33]$.

\section{Acknowledgments}

We are grateful for the technical assistance of Yu-Hsuan Wang and Grants from the Natural Sciences and Engineering Research Council (NSERC), the Canada Research Chairs program, the Canada Foundation for Innovation and the Ontario 
Research Fund. We also appreciate funds from the United States Air Force Office of Scientific Research (AFOSR) under award numbers FA9550-16-1-0109 and FA9550-15-1-0037.

\section{ORCID iDs}

Dong Hyuk Ko (D) https://orcid.org/0000-0002-7840-9995

\section{References}

[1] Corkum P B 1993 Phys. Rev. Lett. 711994

[2] Constant E et al 1999 Phys. Rev. Lett. 821668

[3] Kim H T et al 2003 Phys. Rev. A 67051801

[4] Gariepy G et al 2014 Phys. Rev. Lett. 113153901

[5] Spielmann C et al 1997 Science 278 661-4

[6] López-Martens R et al 2005 Phys. Rev. Lett. 94033001

[7] Fleischer A et al 2014 Nat. Photon. 8 543-9

[8] Kong F et al 2017 Nat. Commun. 814970

[9] Drescher M et al 2002 Nature $\mathbf{4 1 9} 803$

[10] Mairesse Y et al 2003 Science 3021540

[11] Dudovich N et al 2006 Nat. Phys. 434 781-6

[12] Orfanos I et al 2019 APL Photonics 4080901
[13] Corkum P B, Burnett N H and Ivanov M Y 1994 Opt. Lett. 19 $1870-2$

[14] Sansone G et al 2006 Science 314443

[15] Sansone G, Poletto L and Nisoli M 2011 Nat. Photon. 3646

[16] Goulielmakis E et al 2008 Science 3201614

[17] Mashiko H et al 2008 Phys. Rev. Lett. 100103906

[18] Lewenstein M et al 1994 Phys. Rev. A 492117

[19] Lewensten M, Salieres P and L'Huillier A 1995 Phys. Rev. A 524747

[20] Trebino R and Kane D J 1993 J. Opt. Soc. Am. A 101101

[21] Itatani J et al 2002 Phys. Rev. Lett. 88173903

[22] Mairesse Y and Quéré F 2005 Phys. Rev. A 71011401

[23] Kim K T et al 2013 Nat. Phys. 9 159-63

[24] Salieres P et al 2001 Science 292902

[25] Kane D J 2008 J. Opt. Soc. Am. B 25 A120-32

[26] Schmidt B E et al 2010 Appl. Phys. Lett. 96121109

[27] Korobenko A et al 2019 Opt. Express 27 32630-7

[28] Vampa G et al 2014 Phys. Rev. Lett. 113073901

[29] Guan Z, Zhou X and Bian X 2016 Phys. Rev. A 93033852

[30] You Y S et al 2017 Opt. Lett. 42 1816-9

[31] Kim K T et al 2007 Phys. Rev. Lett. 99223904

[32] Vampa G et al 2018 Nat. Photon. 12 465-8

[33] Han S et al 2016 Nat. Commun. 713105 